Research Article

Open Access

Nguyen Huu Hoc*

\title{
Approximate fixed points and fixed points for multi-valued almost $E$-contractions
}

https://doi.org/10.1515/taa-2021-0100

Received 8 August, 2021; accepted 16 November, 2021

Abstract: In this paper, we introduce the concept of multi-valued almost $E$-contractions. We then present some approximate fixed point and fixed point results for such mappings in metric spaces. Our results generalize and improve several well-known results in literature. We also provide several illustrative examples to compare our findings with some earlier results. An application to homotopy theory is given.

Keywords: Approximate fixed points, Fixed points, Multi-valued mappings, E-contractions, Almost contractions, Homotopy theory

MSC 2020: 47H10, 54H 25

\section{Introduction}

Let $(X, d)$ be a metric space. We denote by $P(X)$ the set of all nonempty subsets of $X$, by $C(X)$ the set of all nonempty, closed subsets of $X$ and by $C B(X)$ the set of all nonempty closed bounded subsets of $X$. For a subset $A$ of $X$, we denote by $\bar{A}$ the closure of $A$ and by $\partial A$ the boundary of $A$. For $x \in X$ and $r>0$, we denote by $B(x, r):=\{y \in X: d(y, x)<r\}$ the open ball at $x$ with radius $r$. The distance from $x \in X$ to a subset $A$ of $X$ is defined by

$$
D(x, A):=\inf \{d(x, a): a \in A\} .
$$

For two subsets $A$ and $B$ of $X$, the excess of $A$ over $B$ is defined by

$$
e(A, B):=\sup \{d(a, B): a \in A\} .
$$

The generalized Hausdorff - Pompeiu distance between two subsets $A$ and $B$ of $X$ is defined by

$$
H(A, B):=\max \{e(A, B), e(B, A)\} .
$$

Here, with convention, $D(x, \emptyset)=+\infty$ for $x \in X$ and $e(\emptyset, B)=0$ for $B \subset X$. It is well-known that, for any $x, y \in X$ and $A \subset X$, it holds

$$
D(x, A) \leq d(x, y)+D(y, A) .
$$

The following simple lemma may be known which will be used in the sequel. We present the proof for the reader's convenience.

Lemma 1. Let $(X, d)$ be a metric space, $A$ be a nonempty subset of $X, x \in X$ and $\alpha \in[0,1)$ and $\ell>D(x, A)$. If $D(x, A)>0$, then there exists $a \in A$ such that

$$
d(x, a)<\ell \quad \text { and } \quad \alpha d(x, a)<D(x, A) .
$$

^Corresponding Author: Nguyen Huu Hoc: Faculty of Natural Sciences, Hong Duc University, Thanh Hoa, Vietnam, E-mail: nguyenhuuhoc@hdu.edu.vn 
Proof. It is obvious if $\alpha=0$. If $\alpha>0$, we set

$$
\varepsilon=\min \left\{\ell-D(x, A),\left(\frac{1}{\alpha}-1\right) D(x, A)\right\}>0 .
$$

By the definition of $D(x, A)$, there exists $a \in A$ such that

$$
d(x, a)<D(x, A)+\varepsilon .
$$

By the definition of $\varepsilon$, we obtain (1).

Let $T: X \rightarrow P(X)$ be a multi-valued mapping. We denote by

$$
\operatorname{gph}(T):=\{(x, y) \in X \times X: y \in T(x)\}
$$

the graph of $T$. The multi-valued $T$ mapping is said to be closed if $\operatorname{gph}(T)$ is closed in $X \times X$, i.e., for all sequences $\left\{\left(x_{n}, y_{n}\right)\right\}$ in $\operatorname{gph}(T)$ such that $\left\{\left(x_{n}, y_{n}\right)\right\}$ converges to some $(x, y) \in X \times X$, then $(x, y) \in \operatorname{gph}(T)$. The map $T$ is said to have closed values if $T(x)$ is closed for all $x \in X$. An element $x \in X$ is said to be a fixed point of $T$ if $x \in T(x)$. An orbit of $T$ at a point $x_{0} \in X$ is a sequence $\left\{x_{n}\right\}$ in $X$ such that $x_{n+1} \in T\left(x_{n}\right)$ for all $n \geq 0$. The set of all fixed points of $T$ is denoted by $\operatorname{Fix}(T)$.

A mapping $T: X \rightarrow P(X)$ is said to be a multi-valued contraction or multi-valued $\alpha$-contraction if there exists a constant $\alpha \in[0,1)$ such that

$$
H(T(x), T(y)) \leq \alpha d(x, y), \quad \forall x, y \in X .
$$

Nadler [28] (see also [12]) proved that every multi-valued contraction mapping with closed values in a complete metric space has at least one fixed point. This result is an extension of the famous Banach contraction principle to the context of set-valued mappings. After that, many authors have extended and generalized Nadler's fixed point theorem in different ways. One of the interesting results was given by M. Berinde and V. Berinde in [10] where they introduced the concept of multi-valued weak contractions and proved some fixed point theorems for multi-valued weak contractions.

Definition 1. ([10]) A multi-valued mapping $T: X \rightarrow P(X)$ is said to be multi-valued weak contraction or multi-valued $(\theta, L)$-weak contraction if there exist $\theta \in(0,1)$ and $L \geq 0$ such that

$$
H(T(x), T(y)) \leq \theta d(x, y)+L D(y, T(x)), \quad \forall x, y \in X .
$$

It is proved in [10] that every multi-valued weak contraction in a complete metric space has at least one fixed point. The results in [10] have been extended by many authors. For some extensions and generalizations of the results by Nadler as well as by Berinde - Berinde, we refer the reader to, e.g., [1, 9, 14, 24, 25, 29, 31-33] and references therein.

Recently, Fulga and Proca [15] introduced the notion of $\varphi_{E}$-Geraghty contractions and proved a fixed point theorem.

Definition 2. ([15]) A mapping $T: X \rightarrow X$ is said to be a $\varphi_{E}$-Geraghty contraction if there exists a function $\varphi:[0, \infty) \rightarrow[0,1)$ which satisfies the condition

$$
\lim _{n \rightarrow \infty} \varphi\left(t_{n}\right)=1 \text { implies that } \lim _{n \rightarrow \infty} t_{n}=0,
$$

such that

$$
d(T x, T y) \leq \varepsilon(E(x, y)) E(x, y), \quad \forall x, y \in X,
$$

where $E(x, y)=d(x, y)+|d(x, T x)-d(y, T y)|$.

Later, several researchers have investigated new fixed point results for mappings satisfying generalized conditions involving $E$-contractions (called $P$-contractions in some papers), i.e., conditions which involve the 
expression $E(x, y)=d(x, y)+|d(x, T x)-d(y, T y)|$. For more details, we refer the reader to, e.g., [2-4, 6-8, 16$18,21]$ and references therein. Fixed point results of multi-valued mappings concerning $P$-contractions can be found in $[5,19]$.

It is worth noting that in many practical situations the existence of an approximation solution or an approximate fixed point is more than enough, e.g., approximate Nash equilibria [35], approximate solutions of mathematical programming problems [26]. On the other hand, conditions for ensuring the existence of a fixed point are too strong and may not be satisfied in many real problems. Hence, it is natural to introduce and investigate the theory of $\varepsilon$-approximate fixed points. Starting from the paper by Tijs, Torre and Brânzei [34], many researchers have established various approximate fixed point results for single-valued and multi-valued mappings satisfying different contractive type conditions (see, e.g., $[11,20,27,30]$ and references therein).

Definition 3. ([11, 34]) Let $(X, d)$ be a metric space, $T: X \rightarrow P(X)$ be a multi-valued mapping and $\varepsilon \geq 0$. An element $x^{\star} \in X$ is said to be an $\varepsilon$ - approximate fixed point of $T$ if $D\left(x^{*}, T\left(x^{*}\right)\right) \leq \varepsilon$. The set of all $\varepsilon$-approximate fixed points of $T$ is denoted by $\varepsilon-\operatorname{Fix}(T)$, i.e.,

$$
\varepsilon-\operatorname{Fix}(T)=\{x \in X: D(x, T(x)) \leq \varepsilon\} .
$$

It is evident that $\operatorname{Fix}(T) \subset 0-\operatorname{Fix}(T)$ and $\operatorname{Fix}(T)=0-\operatorname{Fix}(T)$ if $T$ has closed values. Moreover, approximate fixed points may exist under mild conditions in which exact fixed points do not exist. For example, if $T$ is a multi-valued contraction mapping from a metric space $X$ to $P(X)$, then $\varepsilon-\operatorname{Fix}(T) \neq \emptyset$ for every $\varepsilon>0$ (see, e.g.,[27]). However, the set of fixed points of $T$ may be empty when $X$ is not complete. We recall here the following interesting result which is presented in [27].

Theorem 1. ([27]) Let $(X, d)$ be a metric space, $\kappa \in(0,1)$ and let $T: X \rightarrow P(X)$ be a multi-valued $\kappa$-contraction. Then, for every $\varepsilon>0$, the set of $\varepsilon$-approximate fixed points of $T$ is nonempty, i.e., $\varepsilon$-Fix $(T) \neq \emptyset$ and the following estimate is satisfied:

$$
D(x, \varepsilon-\operatorname{Fix}(T)) \leq \frac{1}{1-\kappa} D(x, T(x)), \quad \forall x \in X .
$$

Noting that, by using the results of Theorem 1 and some results about set-convergence in metric spaces, the authors in [27] derive several new results concerning approximate versions of Lim's Lemma, and the celebrated global Lyusternik-Graves Theorem (see, e.g., [13] for more details about these results and their applications).

Inspired by above mentioned results, in this paper, we first introduce the concept of multi-valued almost $E$-contractions and prove an approximate fixed point theorem for such mappings on incomplete metric spaces. This result extends and generalizes several approximate fixed point results in the literature. An approximation of Lim's type result and a data dependence result for approximate fixed point sets of multi-valued almost $E$-contractions are also presented. As corollaries, we also present some fixed point results for multivalued almost $E$-contractions in complete metric spaces. An application to homotopy theory is given as well.

\section{Approximate fixed points for multi-valued almost $E$ - contractions}

We begin this section by introducing the concept of multi-valued almost $E$-contractions.

Definition 4. Let $(X, d)$ be a metric space and $T: X \rightarrow P(X)$ be a multi-valued map. We say that $T$ is a multivalued almost $E$-contraction or multi-valued $(\alpha, \beta, L)$-almost E-contraction if there exist constants $\alpha \in[0,1)$, $\beta \in[0,1]$ and $L \geq 0$ such that

$$
e(T(x), T(y)) \leq \alpha d(x, y)+\beta|D(x, T(x))-D(y, T(y))|+L D(y, T(x)), \quad \forall x, y \in X .
$$

Remark 1. The condition (5) includes several well-known contractive conditions in the literature. In fact, if we let $\beta=0$ in (5), then we get the relation

$$
e(T(x), T(y)) \leq \alpha d(x, y)+L D(y, T(x)), \quad \forall x, y \in X .
$$


Since $e(T(x), T(y)) \leq H(T(x), T(y))$ for all $x, y \in X$, if $T$ is a multi-valued weak contraction, then $T$ satisfies

(6). Thus, a multi-valued weak contraction is a special case of multi-valued almost $E$-contractions.

If $L=0$, then (5) reduces to

$$
e(T(x), T(y)) \leq \alpha d(x, y)+\beta|D(x, T(x))-D(y, T(y))|, \quad \forall x, y \in X .
$$

By the symmetry, we have

$$
e(T(y), T(x)) \leq \alpha d(x, y)+\beta|D(x, T(x))-D(y, T(y))|, \quad \forall x, y \in X .
$$

These relations imply that

$$
H(T(x), T(y)) \leq \alpha d(x, y)+\beta|D(x, T(x))-D(y, T(y))|, \quad \forall x, y \in X .
$$

If $\alpha=\beta \in(0,1)$ in the latter inequality, i.e.,

$$
H(T(x), T(y)) \leq \alpha[d(x, y)+|D(x, T(x))-D(y, T(y))|], \quad \forall x, y \in X,
$$

then we call $T$ a multi-valued E-contraction with constant $\alpha$.

We next give an example of a multi-valued mapping which is a multi-valued $(\alpha, \beta, L)$-almost $E$-contraction but it is not a multi-valued weak contraction.

Example 1. Let $X=\mathbb{R}$ with the usual metric. Define the mapping $T: X \rightarrow P(X)$ by

$$
T(x)=\left\{\begin{array}{ccc}
\{0\} & \text { if } & x \leq 0, \\
{[-2 x,-x]} & \text { if } & x>0
\end{array}\right.
$$

For any $\alpha \in(0,1)$ and $L \geq 0$, let $x=-y>0$, we have

$$
\alpha d(x, y)+L D(y, T(x))=\alpha(x-y)+L .0=2 \alpha x<2 x=H(T(x), T(y)) .
$$

Thus, $T$ is not a multi-valued weak contraction and so $T$ is not a multi-valued contraction.

We next show that $T$ is a multi-valued $(\alpha, \beta, L)$ almos $E$-contraction with $\alpha=\beta=2 / 3$ and $L=1$, i.e.,

$$
e(T(x), T(y)) \leq \frac{2}{3} d(x, y)+\frac{2}{3}|D(x, T(x))-D(y, T(y))|+D(y, T(x)),
$$

for all $x, y \in X$. It is evident that (7) holds for $x, y \in(-\infty, 0]$. We now consider three cases:

Case 1. $x, y \in(0, \infty)$. We may assume $x>y>0$. In this case

$$
e(T(x), T(y)) \leq H(T(x), T(y)=2(x-y),
$$

and the right-hand side of (7) equal to

$$
\frac{2}{3}(x-y)+\frac{2}{3}|2 x-2 y|+D(y, T(x))=2(x-y)+D(y, T(x)) \geq 2(x-y) .
$$

Thus, (7) holds.

Case 2. $x \leq 0$ and $y>0$. In this case, we have $e(T(x), T(y))=y$ and $D(y, T(x))=y$. Thus, (7) holds.

Case 3. $x>0$ and $y \leq 0$. In this case, $e(T(x), T(y))=2 x$ and the right-hand side of (7) equal to

$$
R H S:=\frac{2}{3}(x-y)+\frac{2}{3}|2 x+y|+D(y, T(x)) .
$$

If $y \geq-2 x$, then $R H S=2 x+D(y, T(x)) \geq 2 x=e(T(x), T(y))$. Thus, (7) holds. If $y<-2 x$, then

$$
R H S \geq \frac{2}{3}(x-y)>\frac{2}{3}(x+2 x)=2 x=e(T(x), T(y)) .
$$

Thus, (7) holds. 
The following is the first main result of this paper. It concerns with approximate fixed points of multi-valued almost $E$-contraction mappings.

Theorem 2. Let $(X, d)$ be a metric space and $T: X \rightarrow P(X)$ be a multi-valued $(\alpha, \beta, L)$-almost E-contraction. Then, for every $\varepsilon>0$, the set of $\varepsilon$-approximate fixed points of $T$ is nonempty, i.e., $\varepsilon-F i x(T) \neq \emptyset$ and the following estimate holds true:

$$
D(x, \varepsilon-\operatorname{Fix}(T)) \leq \frac{1+\beta}{1-\alpha} D(x, T(x)),
$$

for all $x \in X$.

Proof. Let $\varepsilon>0, x \in X$ and $\delta>0$ be arbitrary. Set $\ell=D(x, T(x))+\delta$. If $D(x, T(x))=0$, then $x \in \varepsilon-\operatorname{Fix}(T)$. Moreover, $D(x, \varepsilon-\operatorname{Fix}(T))=0=D(x, T(x))$, which implies that (8) holds. Assume that $D(x, T(x))>0$. Then, by Lemma 1 , there exists $x_{1} \in T(x)$ such that

$$
d\left(x, x_{1}\right)<\ell \text { and } \alpha d\left(x, x_{1}\right)<D(x, T(x)) .
$$

By (5), we have

$$
\begin{aligned}
D\left(x_{1}, T\left(x_{1}\right)\right) & \leq e\left(T(x), T\left(x_{1}\right)\right) \\
& \leq \alpha d\left(x, x_{1}\right)+\beta\left|D(x, T(x))-D\left(x_{1}, T\left(x_{1}\right)\right)\right|+L D\left(x_{1}, T(x)\right) \\
& =\alpha d\left(x, x_{1}\right)+\beta\left|D(x, T(x))-D\left(x_{1}, T\left(x_{1}\right)\right)\right| .
\end{aligned}
$$

Assume that $D(x, T(x)) \leq D\left(x_{1}, T\left(x_{1}\right)\right)$. Then, it follows from (9) that

$$
D\left(x_{1}, T\left(x_{1}\right)\right) \leq \alpha d\left(x, x_{1}\right)+\beta D\left(x_{1}, T\left(x_{1}\right)\right)-\beta D(x, T(x))
$$

or, equivalently,

$$
(1-\beta) D\left(x_{1}, T\left(x_{1}\right)\right) \leq \alpha d\left(x, x_{1}\right)-\beta D(x, T(x)) .
$$

Since $D(x, T(x))<D\left(x_{1}, T\left(x_{1}\right)\right)$ and $\alpha d\left(x, x_{1}\right)<D(x, T(x))$, we have

$$
(1-\beta) D(x, T(x))<(1-\beta) D(x, T(x)) .
$$

This is a contradiction. Thus, $D\left(x_{1}, T\left(x_{1}\right)\right)<D(x, T(x))$. Then, one has from (9) that

$$
D\left(x_{1}, T\left(x_{1}\right)\right) \leq \alpha d\left(x, x_{1}\right)+\beta D(x, T(x))-\beta D\left(x_{1}, T\left(x_{1}\right)\right) .
$$

This implies that

$$
D\left(x_{1}, T\left(x_{1}\right)\right) \leq \frac{\alpha}{1+\beta} d\left(x, x_{1}\right)+\frac{\beta}{1+\beta} D(x, T(x))<\frac{\alpha+\beta}{1+\beta} \ell=\theta \ell,
$$

where $\theta=\frac{\alpha+\beta}{1+\beta}$.

If $D\left(x_{1}, T\left(x_{1}\right)\right)=0$, then $x_{1} \in \varepsilon-F i x(T)$. In this case,

$$
D(x, \varepsilon-\operatorname{Fix}(T)) \leq d\left(x, x_{1}\right)<\ell=D(x, T(x))+\delta<\frac{1}{1-\theta} D(x, T(x))+\delta .
$$

Letting $\delta \rightarrow 0^{+}$, we get

$$
D(x, \varepsilon-\operatorname{Fix}(T)) \leq \frac{1}{1-\theta} D(x, T(x)),
$$

i.e., (8) holds. Assume now that $D\left(x_{1}, T\left(x_{1}\right)\right)>0$. Then, again by Lemma 1, there exists $x_{2} \in T\left(x_{1}\right)$ such that

$$
d\left(x_{1}, x_{2}\right)<\theta \ell \text { and } \alpha d\left(x_{1}, x_{2}\right)<D\left(x_{1}, T\left(x_{1}\right)\right) .
$$

Again, by (5), we have

$$
\begin{aligned}
D\left(x_{2}, T\left(x_{2}\right)\right) & \leq e\left(T\left(x_{1}\right), T\left(x_{2}\right)\right) \\
& =\alpha d\left(x_{1}, x_{2}\right)+\beta\left|D\left(x_{1}, T\left(x_{1}\right)\right)-D\left(x_{2}, T\left(x_{2}\right)\right)\right| .
\end{aligned}
$$


If $D\left(x_{1}, T\left(x_{1}\right)\right) \leq D\left(x_{2}, T\left(x_{2}\right)\right)$, then

$$
\begin{aligned}
(1-\beta) D\left(x_{1}, T\left(x_{1}\right)\right. & \leq(1-\beta) D\left(x_{2}, T\left(x_{2}\right)\right) \leq \alpha d\left(x_{1}, x_{2}\right)-\beta D\left(x_{1}, T\left(x_{1}\right)\right. \\
& <D\left(x_{1}, T\left(x_{1}\right)-\beta D\left(x_{1}, T\left(x_{1}\right)\right)=(1-\beta) D\left(x_{1}, T\left(x_{1}\right)\right),\right.
\end{aligned}
$$

which is a contradiction. Thus, $D\left(x_{2}, T\left(x_{2}\right)\right)<D\left(x_{1}, T\left(x_{1}\right)\right)$. By (10),

$$
\begin{aligned}
D\left(x_{2}, T\left(x_{2}\right)\right) & \leq \frac{\alpha}{1+\beta} d\left(x_{1}, x_{2}\right)+\frac{\beta}{1+\beta} D\left(x_{1}, T\left(x_{1}\right)\right) \\
& \leq \frac{\alpha}{1+\beta} \theta \ell+\frac{\beta}{1+\beta} \theta \ell=\theta^{2} \ell .
\end{aligned}
$$

Continuing this progress, we can construct a sequence $\left\{x_{n}\right\}$ in $X$ such that

(a) there exists $k \in \mathbb{N}$ such that $D\left(x_{k}, T\left(x_{k}\right)\right)=0$ and $d\left(x_{i}, x_{i+1}\right)<\theta^{i} \ell$ for all $i=1, \cdots, k-1$, or

(b) for all $n \geq 1$

$$
x_{n+1} \in T\left(x_{n}\right), \quad D\left(x_{n}, T\left(x_{n}\right)\right)<\theta^{n} \ell \text { and } d\left(x_{n}, x_{n+1}\right)<\theta^{n} \ell
$$

If (a) holds, then $x_{k} \in \varepsilon-F i x(T)$ and

$$
\begin{aligned}
D(x, \varepsilon-\operatorname{Fix}(T)) & \leq d\left(x, x_{k}\right) \\
& \leq d\left(x, x_{1}\right)+d\left(x_{1}, x_{2}\right)+\cdots+d\left(x_{k-1}, x_{k}\right) \\
& <\ell+\theta \ell+\cdots+\theta^{k-1} \ell \\
& <\frac{1}{1-\theta} \ell=\frac{1+\beta}{1-\alpha}[D(x, T(x))+\delta] .
\end{aligned}
$$

Letting $\delta \rightarrow 0^{+}$, we get (8).

Assume that (b) happens. Since $\theta \in[0,1), \lim _{n \rightarrow \infty} \theta^{n} \ell=0$. Hence, there exists $n_{0} \in \mathbb{N}$ such that $\theta^{n} \ell<\varepsilon$ for all $n \geq n_{0}$. This means that $D\left(x_{n}, T\left(x_{n}\right)\right)<\varepsilon$ for all $n \geq n_{0}$. Thus, all elements of the sequence $\left\{x_{n}\right\}_{n \geq n_{0}}$ are $\varepsilon$-approximate fixed points of $T$. Moreover, for all $n \in \mathbb{N}$ with $n \geq 2$, we have

$$
\begin{aligned}
d\left(x, x_{n}\right) & \leq d\left(x, x_{1}\right)+d\left(x_{1}, x_{2}\right)+\cdots+d\left(x_{n-1}, x_{n}\right) \\
& <\ell+\theta \ell+\cdots+\theta^{n-1} \ell \\
& \leq \ell\left(1+\theta+\cdots+\theta^{n}+\cdots\right) \\
& =\frac{\ell}{1-\theta}=\frac{1+\beta}{1-\alpha}[D(x, T(x))+\delta] .
\end{aligned}
$$

Since $x_{n} \in \mathcal{E}-\operatorname{Fix}(T)$ for $n$ large enough (i.e., $n \geq n_{0}$ ), we have

$$
D(x, \varepsilon-\operatorname{Fix}(T)) \leq d\left(x, x_{n}\right)<\frac{1+\beta}{1-\alpha}[D(x, T(x))+\delta] .
$$

Letting $\delta \rightarrow 0^{+}$, we get (8). This ends the proof.

In Theorem 2, if we let $\beta=0$ and $L=0$, we obtain Theorem 1 - one of the main results of [27]. If we let $\beta=0$, then we get the following $\varepsilon$-approximate fixed point result for multi-valued weak contraction mappings.

Corollary 1. Let $(X, d)$ be a metric space and $T: X \rightarrow P(X)$ be a multi-valued $(\alpha, L)$-weak contraction. Then, for every $\varepsilon>0$, the set of $\varepsilon$-approximate fixed points of $T$ is nonempty, i.e., $\varepsilon$-Fix $(T) \neq \emptyset$ and the following estimate holds:

$$
D(x, \varepsilon-\operatorname{Fix}(T)) \leq \frac{1}{1-\alpha} D(x, T(x)), \quad \forall x \in X .
$$

We remark that the estimate (12) is new for the case of multi-valued weak contraction mappings. Similarly, if we let $L=0$ in Theorem 2, we obtain the following $\varepsilon$-approximate fixed point result for multi-valued $E$ contraction mappings.

Corollary 2. Let $(X, d)$ be a metric space and $T: X \rightarrow P(X)$ be a multi-valued $E$-contraction with constant $\alpha \in(0,1)$. Then, for every $\varepsilon>0$, the set of $\varepsilon$-approximate fixed points of $T$ is nonempty, i.e., $\varepsilon-\operatorname{Fix}(T) \neq \emptyset$ and the following estimate holds:

$$
D(x, \varepsilon-\operatorname{Fix}(T)) \leq \frac{1+\alpha}{1-\alpha} D(x, T(x)), \quad \forall x \in X .
$$


Example 2. Let $(X, d)$ and $T$ be as in Example 1. For $\varepsilon>0$, we have $\varepsilon-\operatorname{Fix}(T)=[-\varepsilon, \varepsilon / 2]$. Thus, for all $x \in X$, we have

$$
D(x, \varepsilon-\operatorname{Fix}(T))=\left\{\begin{array}{ccc}
-x-\varepsilon & \text { if } & x<-\varepsilon, \\
0 & \text { if } & x \in[-\varepsilon, \varepsilon / 2], \\
x-\varepsilon / 2 & \text { if } & x>\varepsilon / 2,
\end{array}\right.
$$

and

$$
D(x, \varepsilon-\operatorname{Fix}(T)) \leq 5 D(x, T(x))=\frac{1+\beta}{1-\beta} D(x, T(x)) .
$$

Therefore, (8) is verified for this example.

We next provide an example to illustrate Corollary 1.

Example 3. Let $X=\left\{\frac{1}{2^{n}}: n=0,1,2, \cdots\right\}$ with the usual metric $d$. It is evident that $(X, d)$ is not a complete metric space. We define the mapping $T: X \rightarrow P(X)$ as

$$
T(x)=\left\{\begin{array}{cl}
\{1\} & \text { if } \quad x=1, \\
\left\{\frac{1}{2^{n+1}}, \frac{1}{2^{n+2}}\right\} & \text { if } \quad x=\frac{1}{2^{n}}, \quad n=1,2, \cdots
\end{array}\right.
$$

For $n \geq 1$, we have

$$
H\left(T(1), T\left(\frac{1}{2^{n}}\right)\right)=1-\frac{1}{2^{n+2}}, \quad \text { and } \quad d\left(1, \frac{1}{2^{n}}\right)=1-\frac{1}{2^{n}} .
$$

If there exists $\alpha \in(0,1)$ such that

$$
H\left(T(1), T\left(\frac{1}{2^{n}}\right)\right) \leq \alpha d\left(1, \frac{1}{2^{n}}\right),
$$

then

$$
1-\frac{1}{2^{n+2}} \leq \alpha\left(1-\frac{1}{2^{n}}\right)
$$

Letting $n \rightarrow \infty$, we have $1 \leq \alpha$. This is contradicts to $\alpha<1$. Thus, $T$ is not a multi-valued contraction.

We next show that $T$ is a multi-valued $(\alpha, \beta, L)$ almost $E$-contraction with $\alpha=\frac{1}{2}, \beta=0$ and $L=2$, i.e.,

$$
e(T(x), T(y)) \leq \frac{1}{2} d(x, y)+2 D(y, T(x))
$$

for all $x, y \in X$. There are four cases to consider.

Case 1. $x=1$ and $y=\frac{1}{2^{n}}$ with $n \geq 1$. Since

$$
e\left(T(1), T\left(\frac{1}{2^{n}}\right)\right)=1-\frac{1}{2^{n+1}} \quad \text { and } \quad D\left(\frac{1}{2^{n}}, T(1)\right)=1-\frac{1}{2^{n}},
$$

the inequality (14) holds for this case.

Case 2. $x=\frac{1}{2^{n}}$ and $y=1$ with $n \geq 1$. In this case,

$$
e\left(T\left(\frac{1}{2^{n}}\right), T(1)\right)=1-\frac{1}{2^{n+2}} \quad \text { and } \quad D\left(1, T\left(\frac{1}{2^{n}}\right)\right)=1-\frac{1}{2^{n+1}}
$$

Thus, (14) holds.

Case 3. $x=\frac{1}{2^{n}}$ and $y=\frac{1}{2^{n+k}}$ with $n \geq 1$ and $k \geq 1$. In this case,

$$
e\left(T\left(\left(\frac{1}{2^{n}}\right), T\left(\frac{1}{2^{n+k}}\right)\right)=\frac{1}{2^{n+1}}-\frac{1}{2^{n+k+1}}=\left(1-\frac{1}{2^{k}}\right) \frac{1}{2^{n+1}},\right.
$$

and

$$
d\left(\frac{1}{2^{n}}, \frac{1}{2^{n+k}}\right)=\frac{1}{2^{n}}-\frac{1}{2^{n+k}}=2\left(1-\frac{1}{2^{k}}\right) \frac{1}{2^{n+1}}
$$


Thus, (14) holds.

Case 4. $x=\frac{1}{2^{n+k}}$ and $y=\frac{1}{2^{n}}$ with $n \geq 1$ and $k \geq 1$. Since

$$
e\left(T\left(\left(\frac{1}{2^{n+k}}\right), T\left(\frac{1}{2^{n}}\right)\right)=\frac{1}{2^{n+2}}-\frac{1}{2^{n+k+2}}=\left(1-\frac{1}{2^{k}}\right) \frac{1}{2^{n+2}},\right.
$$

and

$$
d\left(\frac{1}{2^{n+k}}, \frac{1}{2^{n}}\right)=4\left(1-\frac{1}{2^{k}}\right) \frac{1}{2^{n+2}},
$$

(14) holds for this case.

For every $\varepsilon>0$, we have $\frac{1}{2^{n}} \in \varepsilon$-Fix $(T)$ for $n=0$ and for $n$ large enough. For $m \geq 1$, we have

$$
\frac{1}{1-\alpha} D\left(\frac{1}{2^{m}}, T\left(\frac{1}{2^{m}}\right)\right)=\frac{1}{2^{m}} \text {. }
$$

For $n$ large enough,

$$
D\left(\frac{1}{2^{m}}, \varepsilon-\operatorname{Fix}(T)\right) \leq\left|\frac{1}{2^{m}}-\frac{1}{2^{n}}\right| \leq \frac{1}{2^{m}}=\frac{1}{1-\alpha} D\left(\frac{1}{2^{m}}, T\left(\frac{1}{2^{m}}\right)\right) .
$$

Therefore, (12) is verified for this example.

The next theorem is an approximate fixed point result of Lim's type for multi-valued almost $E$-contractions. Theorem 3 and its corollary extend [27, Theorem 15] where an estimate similar to (17) for multi-valued contraction mappings was investigated.

Theorem 3. Let $X$ be a metric space and $T_{1}, T_{2}: X \rightarrow P(X)$ be multi-valued $(\alpha, \beta, L)$-almost E-contractions. Then, for every $\varepsilon>0$, the set of $\varepsilon$-approximate fixed points of $T_{i}, i=1,2$, is nonempty. Moreover,

$$
e\left(\varepsilon-\operatorname{Fix}\left(T_{1}\right), \varepsilon-\operatorname{Fix}\left(T_{2}\right)\right) \leq \frac{1+\beta}{1-\alpha} \varepsilon+\frac{1+\beta}{1-\alpha} \sup _{x \in X} e\left(T_{1}(x), T_{2}(x)\right) .
$$

Proof. Let $\varepsilon>0$. By Theorem 2, both $\varepsilon$-Fix $\left(T_{1}\right)$ and $\varepsilon$-Fix $\left(T_{2}\right)$ are nonempty. We now prove (15). Let $x \in X$. By the estimate (8), we have

$$
D\left(x, \varepsilon-\operatorname{Fix}\left(T_{2}\right)\right) \leq \frac{1}{1-\theta} D\left(x, T_{2}(x)\right),
$$

where $\theta=\frac{\alpha+\beta}{1+\beta}$.

For any $\delta>0$, there exists $y \in T_{1}(x)$ such that $d(x, y)<D\left(x, T_{1}(x)\right)+\delta$. From (16),

$$
\begin{aligned}
D\left(x, \varepsilon-\operatorname{Fix}\left(T_{2}\right)\right) & \leq \frac{1}{1-\theta} D\left(x, T_{2}(x)\right) \\
& \leq \frac{1}{1-\theta}\left[d(x, y)+D\left(y, T_{2}(x)\right)\right] \\
& =\frac{1}{1-\theta} d(x, y)+\frac{1}{1-\theta} D\left(y, T_{2}(x)\right) \\
& <\frac{1}{1-\theta}\left[D\left(x, T_{1}(x)\right)+\delta\right]+\frac{1}{1-\theta} D\left(y, T_{2}(x)\right) \\
& \leq \frac{1}{1-\theta} D\left(x, T_{1}(x)\right)+\frac{\delta}{1-\theta}+\frac{1}{1-\theta} e\left(T_{1}(x), T_{2}(x)\right) .
\end{aligned}
$$

Letting $\delta \rightarrow 0^{+}$, we get

$$
D\left(x, \varepsilon-\operatorname{Fix}\left(T_{2}\right)\right) \leq \frac{1}{1-\theta}\left[D\left(x, T_{1}(x)\right)+e\left(T_{1}(x), T_{2}(x)\right)\right] .
$$


Taking supremum in both sides of the latter inequality over $x \in \varepsilon-\operatorname{Fix}\left(T_{1}\right)$, one has

$$
\begin{aligned}
e\left(\varepsilon-\operatorname{Fix}\left(T_{1}\right), \varepsilon-\operatorname{Fix}\left(T_{2}\right)\right) \leq & \sup _{x \in \mathcal{E}-\operatorname{Fix}\left(T_{1}\right)} \frac{1}{1-\theta}\left[D\left(x, T_{1}(x)\right)+e\left(T_{1}(x), T_{2}(x)\right)\right] \\
\leq & \frac{1}{1-\theta} \sup _{x \in \varepsilon-\operatorname{Fix}\left(T_{1}\right)} D\left(x, T_{1}(x)\right) \\
& +\frac{1}{1-\theta} \sup _{x \in \varepsilon-\operatorname{Fix}\left(T_{1}\right)} e\left(T_{1}(x), T_{2}(x)\right) \\
\leq & \frac{1}{1-\theta} \varepsilon+\frac{1}{1-\theta} \sup _{x \in X} e\left(T_{1}(x), T_{2}(x)\right) \\
= & \frac{1+\beta}{1-\alpha} \varepsilon+\frac{1+\beta}{1-\alpha} \sup _{x \in X} e\left(T_{1}(x), T_{2}(x)\right) .
\end{aligned}
$$

This ends the proof.

Corollary 3. Let $X$ be a metric space and $T_{1}, T_{2}: X \rightarrow P(X)$ be multi-valued $(\alpha, \beta, L)$-almost E-contractions. Then, for every $\varepsilon>0$, the set of $\varepsilon$-approximate fixed points of $T_{i}, i=1,2$, is nonempty and

$$
H\left(\varepsilon-\operatorname{Fix}\left(T_{1}\right), \varepsilon-\operatorname{Fix}\left(T_{2}\right)\right) \leq \frac{1+\beta}{1-\alpha} \varepsilon+\frac{1+\beta}{1-\alpha} \sup _{x \in X} H\left(T_{1}(x), T_{2}(x)\right) .
$$

Proof. Let $\varepsilon>0$. By Theorem 3, we have

$$
e\left(\varepsilon-\operatorname{Fix}\left(T_{1}\right), \varepsilon-\operatorname{Fix}\left(T_{2}\right)\right) \leq \frac{1+\beta}{1-\alpha} \varepsilon+\frac{1+\beta}{1-\alpha} \sup _{x \in X} e\left(T_{1}(x), T_{2}(x)\right) .
$$

Since

$$
e\left(T_{1}(x), T_{2}(x)\right) \leq H\left(T_{1}(x), T_{2}(x)\right), \quad \forall x \in X,
$$

we have

$$
e\left(\varepsilon-\operatorname{Fix}\left(T_{1}\right), \varepsilon-\operatorname{Fix}\left(T_{2}\right)\right) \leq \frac{1+\beta}{1-\alpha} \varepsilon+\frac{1+\beta}{1-\alpha} \sup _{x \in X} H\left(T_{1}(x), T_{2}(x)\right) .
$$

Similarly, one has

$$
e\left(\varepsilon-\operatorname{Fix}\left(T_{2}\right), \varepsilon-\operatorname{Fix}\left(T_{1}\right)\right) \leq \frac{1+\beta}{1-\alpha} \varepsilon+\frac{1+\beta}{1-\alpha} \sup _{x \in X} H\left(T_{1}(x), T_{2}(x)\right) .
$$

It follows from (18) and (19) that

$$
H\left(\varepsilon-\operatorname{Fix}\left(T_{2}\right), \varepsilon-\operatorname{Fix}\left(T_{1}\right)\right) \leq \frac{1+\beta}{1-\alpha} \varepsilon+\frac{1+\beta}{1-\alpha} \sup _{x \in X} H\left(T_{1}(x), T_{2}(x)\right) .
$$

The proof is complete.

From the proof of Theorem 3, we can also derive a data dependence result for the $\varepsilon$-approximate fixed point set of a multi-valued almost $E$-contraction.

Theorem 4. Let $(X, d)$ be a metric space, $\varepsilon>0$ and $T, S: X \rightarrow P(X)$ be such that

(a) $T$ is a multi-valued $(\alpha, \beta, L)$-almost E-contraction;

(b) $\varepsilon-\operatorname{Fix}(S) \neq \emptyset$;

(c) there exists $\eta>0$ such that $e(T(x), S(x)) \leq \eta$ for all $x \in X$.

Then,

$$
e(\varepsilon-\operatorname{Fix}(S), \varepsilon-\operatorname{Fix}(T)) \leq \frac{1+\beta}{1-\alpha}(\varepsilon+\eta)
$$

Proof. As in the proof of Theorem 3, replacing $\varepsilon$-Fix $\left(T_{2}\right)$ by $\varepsilon-F i x(T), T_{1}$ by $S$ and taking supremum over $x \in \varepsilon-F i x(S)$, we get

$$
e(\varepsilon-\operatorname{Fix}(S), \varepsilon-\operatorname{Fix}(T)) \leq \frac{1+\beta}{1-\alpha} \varepsilon+\frac{1+\beta}{1-\alpha} \sup _{x \in X} e(S(x), T(x)) .
$$

Using (c), we obtain the desired inequality. The proof is complete. 


\section{Fixed points results for multi-valued almost $E$-contractions and application}

In this section, we present some fixed point results for multi-valued almost $E$-contraction mappings.An application to homotopy theory is also given.

\subsection{Fixed points results for multi-valued almost $E$-contractions}

Our first fixed point theorem is stated as follows.

Theorem 5. Let $(X, d)$ be a metric space and $T: X \rightarrow C(X)$ be a multi-valued $(\alpha, \beta, L)$-almost E-contraction. If one of the following condition holds:

(i) the graph of $T$ is complete;

(ii) $X$ is complete, $T$ has closed values and $x \rightarrow D(x, T(x))$ is lower semi-continuous;

(iii) $X$ is complete, $T$ has closed values and $\beta<1$;

then

(a) $\operatorname{Fix}(T)$ is nonempty.

(b) For any $x_{0} \in X$, there exists an orbit $\left\{x_{n}\right\}_{n=0}^{\infty}$ of $T$ at $x_{0}$ converging to a fixed point $\bar{x}$ of $T$ such that

$$
d\left(x_{n}, \bar{x}\right) \leq\left(\frac{\alpha+\beta}{1+\beta}\right)^{n} \frac{1+\beta}{1-\alpha} d\left(x_{0}, x_{1}\right), \quad n=0,1,2, \cdots
$$

(c) The following estimate is satisfied:

$$
D\left(x_{0}, \operatorname{Fix}(T)\right) \leq \frac{1+\beta}{1-\alpha} D\left(x_{0}, T\left(x_{0}\right)\right), \quad \forall x_{0} \in X .
$$

Proof. Let $x \in X$ and $\ell$ be such that $D(x, T(x))<\ell<D(x, T(x))+1$. Set

$$
\theta=\frac{\alpha+\beta}{1+\beta} .
$$

As in the proof of Theorem 2, we can construct a sequence $\left\{x_{n}\right\}$ in $X$ with $x_{0}=x$ satisfying either

(I) there exists a nonnegative integer $n_{0}$ such that $D\left(x_{n_{0}} T\left(x_{n_{0}}\right)\right)=0$ and

$$
x_{n+1} \in T\left(x_{n}\right), D\left(x_{n}, T\left(x_{n}\right)\right)<\theta^{n} \ell \text {, and } d\left(x_{n}, x_{n+1}\right)<\theta^{n} \ell,
$$

for all $n=0, \cdots, n_{0}-1$; or

(II) for all $n \geq 0$

$$
x_{n+1} \in T\left(x_{n}\right), D\left(x_{n}, T\left(x_{n}\right)\right)<\theta^{n} \ell \text {, and } d\left(x_{n}, x_{n+1}\right)<\theta^{n} \ell .
$$

Assume that (I) holds. Since $D\left(x_{n_{0}} T\left(x_{n_{0}}\right)\right)=0$ and $T$ has closed values, $x_{n_{0}} \in T\left(x_{n_{0}}\right)$. In this case, we can put $x_{n}=x_{n_{0}}$ for all $n \geq n_{0}$ and the sequence $\left\{x_{n}\right\}$ satisfies (II). That is, we can always construct an orbit $\left\{x_{n}\right\}$ of $T$ at $x_{0}$ satisfies (22) for all $n \geq 0$. For $m>n$, we have

$$
d\left(x_{n}, x_{m}\right) \leq \sum_{i=n}^{m-1} d\left(x_{i}, x_{i+1}\right)<\sum_{i=n}^{m-1} \theta^{i} \ell \leq \frac{\theta^{n}}{1-\theta} \ell .
$$

Since $\theta \in(0,1)$, it follows from (23) that $\left\{x_{n}\right\}$ is a Cauchy sequence.

Assume that (i) holds. Since the graph of $T$ is complete and $x_{n+1} \in T\left(x_{n}\right),\left\{x_{n}\right\}$ converges to some $\bar{x} \in X$ with $(\bar{x}, \bar{x}) \in \operatorname{gph}(T)$. Thus, $\bar{x}$ is a fixed point of $T$ and so $\operatorname{Fix}(T) \neq \emptyset$.

Suppose now that (ii) holds. Since $X$ is complete, there is some $\bar{x} \in X$ such that $x_{n} \rightarrow \bar{x}$ as $n \rightarrow \infty$. By the lower semicontinuity of $x \rightarrow D(x, T(x))$, we have

$$
0 \leq D(\bar{x}, T(\bar{x})) \leq \liminf _{n \rightarrow \infty} D\left(x_{n}, T\left(x_{n}\right)\right) \leq \liminf _{n \rightarrow \infty} d\left(x_{n}, x_{n+1}\right)=0 .
$$


Thus, $D(\bar{x}, T(\bar{x}))=0$. The closedness of $T(\bar{x}))$ implies that $\bar{x} \in T(\bar{x})$, i.e., $\operatorname{Fix}(T) \neq \emptyset$.

Let assume now that (iii) holds. Since $X$ is complete, there exists $\bar{x} \in X$ such that $\lim _{n \rightarrow \infty} x_{n}=\bar{x}$. Since $x_{n+1} \in T\left(x_{n}\right)$, we have $\lim _{n \rightarrow \infty} D\left(x_{n}, T\left(x_{n}\right)\right)=0$. Moreover,

$$
\begin{aligned}
D(\bar{x}, T(\bar{x})) \leq & d\left(\bar{x}, x_{n+1}\right)+D\left(x_{n+1}, T(\bar{x})\right) \\
\leq & d\left(\bar{x}, x_{n+1}\right)+e\left(T\left(x_{n}\right), T(\bar{x})\right) \\
\leq & d\left(\bar{x}, x_{n+1}\right)+\alpha d\left(x_{n}, \bar{x}\right) \\
& \left.+\beta \mid D\left(x_{n}\right), T\left(x_{n}\right)\right)-D(\bar{x}, T(\bar{x})) \mid+L D\left(\bar{x}, T\left(x_{n}\right)\right) \\
\leq & \left.d\left(\bar{x}, x_{n+1}\right)+\alpha d\left(x_{n}, \bar{x}\right)+\beta \mid D\left(x_{n}\right), T\left(x_{n}\right)\right)-D(\bar{x}, T(\bar{x})) \mid \\
& +L D\left(\bar{x}, x_{n}\right)+D\left(x_{n}, T\left(x_{n}\right)\right) .
\end{aligned}
$$

Letting $n \rightarrow \infty$, we get $D(\bar{x}, T(\bar{x})) \leq \beta D(\bar{x}, T(\bar{x}))$ which implies $D(\bar{x}, T(\bar{x}))=0$. By the closedness of $T(\bar{x})$, we obtain $\bar{x} \in T(\bar{x})$, i.e., $\operatorname{Fix}(T) \neq \emptyset$.

Since (23) holds for any $\ell>D\left(x_{0}, T\left(x_{0}\right)\right)$, we have, for all $m>n$ that

$$
d\left(x_{n}, x_{m}\right) \leq \frac{\theta^{n}}{1-\theta} D\left(x_{0}, T\left(x_{0}\right)\right) .
$$

Letting $m \rightarrow \infty$, we get

$$
\left.d\left(x_{n}, \bar{x}\right)\right) \leq \frac{\theta^{n}}{1-\theta} D\left(x_{0}, T\left(x_{0}\right)\right) \leq\left(\frac{\alpha+\beta}{1+\beta}\right)^{n} \frac{1+\beta}{1-\alpha} d\left(x_{0}, x_{1}\right) .
$$

Thus, (20) holds. Now, in (24), letting $n=0$, we have

$$
d\left(x, x_{m}\right) \leq \frac{1}{1-\theta} D\left(x_{0}, T\left(x_{0}\right)\right)=\frac{1+\beta}{1-\alpha} D\left(x_{0}, T\left(x_{0}\right)\right) .
$$

Letting $m \rightarrow \infty$, we get

$$
D(x, \operatorname{Fix}(T)) \leq d(x, \bar{x}) \leq \frac{1+\beta}{1-\alpha} D\left(x_{0}, T\left(x_{0}\right)\right) .
$$

Thus, (21) holds. This ends the proof.

Remark 2. (i) Theorem 5 covers [27, Corollary 3], [10, Theorem 3] and Nadler's theorem [28].

(ii) The estimate (21) can be obtained from the estimate (8) by using some results about set-convergence as in [27].

Example 4. Let $(X, d)$ and $T$ be as in Example 1. Then, all conditions of Theorem 5 are satisfied. We have $\operatorname{Fix}(T)=\{0\}$. For all $x \in X$, we have $D(x, \operatorname{Fix}(T))=|x|$ and

$$
D(x, T(x))=\left\{\begin{array}{lll}
-x & \text { if } \quad x \leq 0 \\
2 x & \text { if } \quad x>0
\end{array}\right.
$$

Thus,

$$
D(x, \operatorname{Fix}(T)) \leq 2 D(x, T(x)) \leq 5 D(x, T(x))=\frac{1+\beta}{1-\beta} D(x, T(x))
$$

for all $x \in X$, i.e., (21) is verified.

Example 5. Let $(X, d)$ and $T$ be as in Example 3. That is, $T$ is a multi-valued $(\alpha, \beta, L)$-almost $E$-contraction with $\alpha=\frac{1}{2}, \beta=0$ and $L=2$. We have $\operatorname{Fix}(T)=\{1\}$ and for $n \geq 1$,

$$
D\left(\frac{1}{2^{n}}, \operatorname{Fix}(T)\right)=1-\frac{1}{2^{n}} \rightarrow 1 \quad \text { as } n \rightarrow \infty,
$$

whereas

$$
D\left(\frac{1}{2^{n}}, T\left(\frac{1}{2^{n}}\right)\right)=\frac{1}{2^{n+1}} \rightarrow 0 \quad \text { as } \quad n \rightarrow \infty .
$$


Thus, for $n$ large enough, we have

$$
D\left(\frac{1}{2^{n}}, \operatorname{Fix}(T)\right)>\frac{1+\beta}{1-\alpha} D\left(\frac{1}{2^{n}}, T\left(\frac{1}{2^{n}}\right)\right)=2 D\left(\frac{1}{2^{n}}, T\left(\frac{1}{2^{n}}\right)\right) .
$$

So (21) does not hold for this example. This happens since $(X, d)$ and the graph of $T$ are not complete.

Using the estimate (21) and arguing as in the proof of Theorem 3 and Corollary 3, one can prove the following result.

Theorem 6. Let $X$ be a metric space and $T_{1}, T_{2}: X \rightarrow C(X)$ be multi-valued $(\alpha, \beta, L)$-almost E-contractions. Assume that one of the following conditions holds

(a) the graph of $T_{i}, i=1,2$, is complete;

(b) $X$ is complete and $x \rightarrow D\left(x, T_{i}(x)\right), i=1,2$, is lower semi-continuous;

(c) $X$ is complete and $\beta<1$.

Then, $\operatorname{Fix}\left(T_{1}\right) \neq \emptyset, \operatorname{Fix}\left(T_{2}\right) \neq \emptyset$ and

$$
e\left(\operatorname{Fix}\left(T_{1}\right), \operatorname{Fix}\left(T_{2}\right)\right) \leq \frac{1+\beta}{1-\alpha} \sup _{x \in X} e\left(T_{1}(x), T_{2}(x)\right)
$$

and

$$
H\left(\operatorname{Fix}\left(T_{1}\right), \operatorname{Fix}\left(T_{2}\right)\right) \leq \frac{1+\beta}{1-\alpha} \sup _{x \in X} H\left(T_{1}(x), T_{2}(x)\right) .
$$

The following corollary covers [25, Theorem 1$]$.

Corollary 4. Let $(X, d)$ be a metric space and $\left\{T_{n}\right\}_{n \geq 0}$ be a sequence of multi-valued $(\alpha, \beta, L)$-almost Econtractions from $X$ into $C(X)$. Assume that one of the following conditions holds:

(a) the graph of $T_{n}, n=0,1,2, \cdots$, is complete;

(b) $X$ is complete and $x \rightarrow D\left(x, T_{n}(x)\right)$ is lower semi-continuous for all $n=0,1,2, \cdots$;

(c) $X$ is complete and $\beta<1$.

If $\lim _{n \rightarrow \infty} H\left(T_{n}(x), T_{0}(x)\right)=0$ uniformly for all $x \in X$, then

$$
\lim _{n \rightarrow \infty} H\left(\operatorname{Fix}\left(T_{n}\right), \operatorname{Fix}\left(T_{0}\right)\right)=0 .
$$

Proof. By Theorem 5, Fix $\left(T_{n}\right) \neq \emptyset$ for all $n=0,1,2 \cdots$. Since

$$
\lim _{n \rightarrow \infty} H\left(T_{n}(x), T_{0}(x)\right)=0
$$

uniformly for all $x \in X$, for any $\varepsilon>0$, there exists $N \in \mathbb{N}$ such that

$$
\sup _{x \in X} H\left(T_{n}(x), T_{0}(x)\right)<(1-\theta) \varepsilon,
$$

for all $n \geq N$, where $\theta=(\alpha+\beta) /(1+\beta)$. Using the estimate (26), we have

$$
H\left(\operatorname{Fix}\left(T_{n}\right), \operatorname{Fix}\left(T_{0}\right)\right)<\varepsilon,
$$

for all $n \geq N$. This implies that $\lim _{n \rightarrow \infty} H\left(\operatorname{Fix}\left(T_{n}\right)\right.$, $\operatorname{Fix}\left(T_{0}\right)=0$. The proof is complete.

One can also give the local versions of above results. For instance, we have the following local fixed point theorem for multi-valued almost $E$-contractions.

Theorem 7. Let $(X, d)$ be a complete metric space, $x_{0} \in X, r>0$ and $T: X \rightarrow C(X)$. Assume that there exist $\alpha \in[0,1), \beta \in[0,1)$ and $L \geq 0$ such that

$$
e(T(x), T(y)) \leq \alpha d(x, y)+\beta|D(x, T(x))-D(y, T(y))|+L D(y, T(x)),
$$


for all $x, y \in B\left(x_{0}, r\right)$. If

$$
D\left(x_{0}, T\left(x_{0}\right)\right)<\frac{(1-\alpha) r}{1+\beta},
$$

then $T$ has a fixed point in $B\left(x_{0}, r\right)$ and

$$
D\left(x_{0}, \operatorname{Fix}(T)\right) \leq \frac{1+\beta}{1-\alpha} D\left(x_{0}, T\left(x_{0}\right)\right) .
$$

Proof. Set $\theta=\frac{\alpha+\beta}{1+\beta} \in[0,1)$. Let $\ell$ be such that

$$
D\left(x_{0}, T\left(x_{0}\right)\right)<\ell<\frac{(1-\alpha) r}{1+\beta}=(1-\theta) r .
$$

If $D\left(x_{0}, T\left(x_{0}\right)\right)$, then $x_{0}$ is a fixed point of $T$ in $B\left(x_{0}, r\right)$ and the proof is finished. If $D\left(x_{0}, T\left(x_{0}\right)\right)>0$, then there exists $x_{1} \in T\left(x_{0}\right)$ such that $d\left(x_{0}, x_{1}\right)<\ell$ and $\alpha d\left(x_{0}, x_{1}\right)<D\left(x_{0}, T\left(x_{0}\right)\right)$. Since $d\left(x_{0}, x_{1}\right)<\ell<r, x_{1} \in B\left(x_{0}, r\right)$. Using (27) and arguing as in the proof of Theorem 2, we have $D\left(x_{1}, T\left(x_{1}\right)\right)<\theta \ell$. Again, if $D\left(x_{1}, T\left(x_{1}\right)\right)=0$, then $x_{1} \in \operatorname{Fix}(T)$ and $D\left(x_{0}, \operatorname{Fix}(T)\right) \leq d\left(x_{0}, x_{1}\right)<\ell$. Since $\ell>D\left(x_{0}, T\left(x_{0}\right)\right)$ is arbitrary, the latter inequality implies that

$$
D\left(x_{0}, \operatorname{Fix}(T)\right) \leq D\left(x_{0}, T\left(x_{0}\right)\right) \leq \frac{1+\beta}{1-\alpha} D\left(x_{0}, T\left(x_{0}\right)\right),
$$

i.e., (28) holds. Otherwise, there exists $x_{2} \in T\left(x_{1}\right)$ such that $d\left(x_{1}, x_{2}\right)<\theta \ell$ and $\alpha d\left(x_{1}, x_{2}\right)<D\left(x_{1}, T\left(x_{1}\right)\right)$. In this case,

$$
d\left(x_{0}, x_{2}\right) \leq d\left(x_{0}, x_{1}\right)+d\left(x_{1}, x_{2}\right)<\ell+\theta \ell<\left(1-\theta^{2}\right) r \leq r,
$$

i.e., $x_{2} \in B\left(x_{0}, r\right)$. Continuing this progress, as arguing in the proof of Theorem 5 , we can construct an orbit $\left\{x_{n}\right\}$ of $T$ at $x_{0}$ such that

$$
x_{n+1} \in T\left(x_{n}\right) \text { and } d\left(x_{n}, x_{n+1}\right)<\theta^{n} \ell
$$

and moreover $x_{n} \in B\left(x_{0}, r\right)$ for all $n$. Again, as in the proof of Theorem 5, $\left\{x_{n}\right\}$ is a Cauchy sequence and it converges to some $\bar{x} \in X$ satisfying

$$
d\left(x_{n}, \bar{x}\right) \leq \frac{\theta^{n}}{1-\theta} D\left(x_{0}, T\left(x_{0}\right)\right),
$$

for all $n \geq 0$. In particular,

$$
d\left(x_{0}, \bar{x}\right) \leq \frac{1}{1-\theta} D\left(x_{0}, T\left(x_{0}\right)\right)<\frac{1}{1-\theta}(1-\theta) r=r,
$$

i.e., $\bar{x} \in B\left(x_{0}, r\right)$. The latter inequality also implies that (28) holds. This ends the proof.

\subsection{An application to homotopy theory}

To conclude this paper, we give an application of our results to homotopy theory. Similar results for other general contractive multi-valued mappings can be found in, e.g., $[1,9,24,29]$ and references therein.

Theorem 8. Let $(X, d)$ be a complete metric space, $\Omega$ be an open subset of $X$ and $F: \bar{\Omega} \times[0,1] \rightarrow C(X)$. Assume that the following conditions are satisfied:

(i) $x \notin F(x, t)$ for $x \in \partial \Omega$ and $t \in[0,1]$;

(ii) there exist $\alpha, \beta \in[0,1)$ and $L \geq 0$ such that

$$
e(F(x, t), F(y, t)) \leq \alpha d(x, y)+\beta|D(x, F(x, t))-D(y, F(y, t))|+L D(y, F(x, t))
$$

for all $x, y \in \bar{\Omega}, t \in[0,1]$;

(iii) for any $\varepsilon>0$, there exists $\delta>0$ such that when $t, s \in[0,1]$ with $|t-s|<\delta$, then $e(F(x, t), F(x, s))<\varepsilon$ for all $x \in \bar{\Omega}$;

(iv) $\inf \{D(x, F(x, t)): x \in \bar{\Omega}, t \in[0,1]\}>0$. 
If $F(\cdot, 0)$ has a fixed point, then $F(\cdot, t)$ has a fixed point in $\Omega$ for all $t \in[0,1]$.

Proof. Assume that $F(\cdot, 0)$ has a fixed point. We set

$$
\Lambda=\{t \in[0,1]: x \in F(x, t) \text { for some } x \in \Omega\} .
$$

Since $F(\cdot, 0)$ has a fixed point and $x \notin F(x, t)$ for $x \in \partial \Omega$ and $t \in[0,1], F(\cdot, 0)$ has a fixed point in $\Omega$. Thus, $0 \in \Lambda$, i.e., $\Lambda$ is nonempty. We shall show that $\Lambda$ is both closed and open in [0,1].

We first show that $\Lambda$ is closed in $[0,1]$. Let $\left\{t_{k}\right\}$ be a sequence in $\Lambda$ and it converges to some $\lambda \in[0,1]$. We are going to show that $\lambda \in \Lambda$. Since $\left\{t_{k}\right\} \subset \Lambda$, there exists a sequence $\left\{x_{k}\right\}$ in $\Omega$ such that $x_{k} \in F\left(x_{k}, t_{k}\right)$ for all $k$. We claim that

$$
\inf \left\{D\left(x_{k}, \partial \Omega\right): k \geq 1\right\}>0 .
$$

Assume that the claim is not true. Then, we may assume (after taking subsequence of $\left\{x_{k}\right\}$ ) that there exists a sequence $\left\{y_{k}\right\}$ in $\partial \Omega$ such that

$$
d\left(x_{k}, y_{k}\right)<\frac{1}{k}, \quad \forall k \geq 1
$$

This together with (iv) yields

$$
0<\inf \{D(x, F(x, t)): x \in \partial \Omega, t \in[0,1]\} \leq \liminf _{k \rightarrow \infty} D\left(y_{k}, F\left(y_{k}, t_{k}\right)\right) .
$$

Now, using (ii), we have for all $k$ that

$$
\begin{aligned}
D\left(y_{k}, F\left(y_{k}, t_{k}\right)\right) \leq & d\left(y_{k}, x_{k}\right)+D\left(x_{k}, F\left(y_{k}, t_{k}\right)\right) \\
< & \frac{1}{k}+e\left(F\left(x_{k}, t_{k}\right), F\left(y_{k}, t_{k}\right)\right) \\
\leq & \frac{1}{k}+\alpha d\left(x_{k}, y_{k}\right)+\beta\left|D\left(x_{k}, F\left(x_{k}, t_{k}\right)\right)-D\left(y_{k}, F\left(y_{k}, t_{k}\right)\right)\right| \\
& +L D\left(y_{k}, F\left(x_{k}, t_{k}\right)\right) \\
\leq & \frac{1+\alpha}{k}+\beta D\left(y_{k}, F\left(y_{k}, t_{k}\right)\right)+L D\left(y_{k}, F\left(x_{k}, t_{k}\right)\right) \\
\leq & \frac{1+\alpha}{k}+\beta D\left(y_{k}, F\left(y_{k}, t_{k}\right)\right)+L d\left(y_{k}, x_{k}\right)+L D\left(x_{k}, F\left(x_{k}, t_{k}\right)\right) \\
\leq & \frac{1+\alpha+L}{k}+\beta D\left(y_{k}, F\left(y_{k}, t_{k}\right)\right) .
\end{aligned}
$$

This implies that

$$
D\left(y_{k}, F\left(y_{k}, t_{k}\right)\right) \leq \frac{1+\alpha+L}{(1-\beta) k} .
$$

Taking the limit as $k \rightarrow \infty$, we get

$$
\liminf _{k \rightarrow \infty} D\left(y_{k}, F\left(y_{k}, t_{k}\right)\right) \leq 0
$$

which contradicts (30). Thus, (29) holds. As a results, there exists $r>0$ such that $B\left(x_{k}, r\right) \subset \Omega$ for all $k$. Moreover, since $t_{k} \rightarrow \lambda$ as $k \rightarrow \infty$, by (iii), there exists a natural number $k_{0}$ such that

$$
D\left(x_{k_{0}}, F\left(x_{k_{0}}, \lambda\right)\right) \leq e\left(F\left(x_{k_{0}}, t_{k_{0}}\right), F\left(x_{k_{0}}, \lambda\right)\right)<\frac{(1-\alpha) r}{1+\beta} .
$$

Applying Theorem 7, we conclude that $F(\cdot, \lambda)$ has a fixed point $\bar{x} \in B\left(x_{k_{0}}, r\right) \subset \Omega$, i.e., there exists $\bar{x} \in \Omega$ such that $\bar{x} \in F(\bar{x}, \lambda)$. This implies that $\lambda \in \Lambda$. Thus, $\Lambda$ is closed in $[0,1]$.

We now show that $\Lambda$ is open in $[0,1]$. Let $t_{0} \in \Lambda$. Then there exists $x_{0} \in \Omega$ such that $x_{0} \in F\left(x_{0}, t_{0}\right)$. Since $\Omega$ is open, there exists $r>0$ such that $B\left(x_{0}, r\right) \subset \Omega$. By (iii), for $\varepsilon=\frac{(1-\alpha) r}{1+\beta}$, there exists $\delta=\delta(\varepsilon)>0$ such that

$$
D\left(x_{0}, F\left(x_{0}, t\right)\right) \leq e\left(F\left(x_{0}, t_{0}\right), F\left(x_{0}, t\right)\right)<\frac{(1-\alpha) r}{1+\beta},
$$


for all $t \in[0,1]$ satisfying $\left|t-t_{0}\right|<\delta$. Hence, by Theorem 7 , for each $t \in[0,1]$ with $\left|t-t_{0}\right|<\delta, F(\cdot, t)$ has a fixed point in $B\left(x_{0}, r\right) \subset \Omega$. That is, for each $t \in[0,1]$ with $\left|t-t_{0}\right|<\delta$, there exists $x_{t} \in \Omega$ such that $x_{t} \in F\left(x_{t}, t\right)$. This means that

$$
\left\{t \in[0,1]:\left|t-t_{0}\right|<\delta\right\} \subset \Lambda .
$$

Thus, $\Lambda$ is open in $[0,1]$. Since $\Lambda$ is both closed and open in $[0,1]$ and $[0,1]$ is connected, we conclude that $\Lambda=[0,1]$. This ends the proof.

Acknowledgments: The author would like to thank the two anonymous referees for valuable comments and suggestions which helped to improve the manuscript.

Conflict of interest: Author state no conflict of interest.

\section{References}

[1] Agarwal, R.P., Dshalalow, J. and O’Regan, D. Fixed point and homotopy results for generalized contractive maps of Reich type, Appl. Anal. 82 (2003) 329-350.

[2] Alqahtani, B., Fulga, A. and Karapınar, E. Sehgal Type Contractions on b-Metric Space, Symmetry, 10(11) (2018) 560.

[3] Alqahtani, B., Fulga, A. and Karapınar, E. A short note on the common fixed points of the Geraghty contraction of type $E_{S, T}$, Demonstr. Math. 51 (2018) 233-240.

[4] Altun, I., Durmaz, G. and Olgun, M. P-contractive mappings on metric spaces, J. Nonlinear Funct. Anal. 2018 (2018) Article ID 43.

[5] Altun, I., Hancer, H.A. and Erduran, A. Fixed point results for single valued and set valued P-contractions and application to second order boundary value problems, Carpathian J. Math, 36 (2020) 205 - 214.

[6] Altun, I., Aslantas, M. and Sahin, H. Best proximity point results for p-proximal contractions, Acta Math. Hungar. 162 (2020) 393-402.

[7] Aslantas, M., Sahin, H. and Altun, I. Best proximity point theorems for cyclic p-contractions with some consequences and applications, Nonlinear Anal - Model., 26 (2021) 113-129.

[8] Aydi, H., Felhi, A., Karapınar, E., Alrubaish, H. and Alshammari, M. Fixed points for $\alpha$ - $\beta_{E}$-Geraghty contractions on $b$-metric spaces and applications to matrix equations, Filomat, 33(12) (2019) 3737-3750.

[9] Beg, I. and Pathak, H.K. A variant of Nadler's theorem on weak partial metric spaces with application to a homotopy result, Vietnam J. Math. 46 (2018) 693-706.

[10] Berinde, M. and Berinde, V. On a general class of multi-valued weakly Picard mappings, J. Math. Anal. Appl. 326 (2007) 772-782.

[11] Brânzei, R., Morgan, J., Scalzo, V. and Tijs, S. Approximate fixed point theorems in Banach spaces with applications in game theory, J. Math. Anal. Appl. 285 (2003) 619-628.

[12] Covitz, H. and Nadler jr., S. B. Multi-valued contraction mappings in generalized metric spaces, Israel J. Math. 8 (1970) 5-11.

[13] Dontchev, A.L. and Rockafellar, R.T. Implicit Functions and Solution Mappings, 2nd edn. Heidelberg, New York, 2014.

[14] Feng, Y. and Liu, S. Fixed point theorems for multi-valued contractive mappings and multi-valued Caristi type mappings, J. Math. Anal. Appl. 317 (2006) 103-112.

[15] Fulga, A. and Proca, A. M. Fixed points for $\varphi_{E}$-Geraghty contractions, J. Nonlinear Sci. Appl. 10 (2017) 5125-5131.

[16] Fulga, A. and Proca, A. M. A new generalization of Wardowski fixed point theorem in complete metric spaces, Advances in the Theory of Nonlinear Analysis and its Applications, 1 (2017) 57-63.

[17] Fulga, A. and Karapinar, E. Revisiting of some outstanding metric fixed point theorems via E-contraction, An. St. Univ. Ovidius Constanta, Ser. Mat. 26 (2018) 73-97.

[18] Fulga, A. and Karapınar, E. Some Results on S-Contractions of Type E, Mathematics, 6(10) (2018), 195.

[19] Hancer, H.A. On multivalued P-contractive mappings that belongs to class of weakly Picard operators, Fixed Point Theory, 22 (2021) 663-670.

[20] Hussain, N., Amini-Harandi, A. and Cho, Y. J. Approximate endpoints for set-valued contractions in metric spaces, Fixed Point Theory Appl. 2010, Art. ID 614867, 13 pp.

[21] Hoc, N.H., Hung, H.V. and Ngoc, L.T.P. Discussions on the fixed points of Suzuki-Edelstein E-contractions. Rend. Circ. Mat. Palermo, II. Ser (2021). https://doi.org/10.1007/s12215-021-00637-3

[22] Karapinar, E., Fulga, A. and Aydi, H. Study on Pata E-contractions, Adv. Differ. Equ. 2020, 539 (2020)

[23] Karapınar, E., Fulga, A. and Petrusel, A. On Istratescu type contractions in b-metric spaces, Mathematics, 8(3) (2020), 388.

[24] Lazar, T., O'Regan, D. and Petrusel, A. Fixed points and homotopy results for Ćirić type multivalued operators on a set with two metrics, Bull. Korean Math. Soc. 45 (2008) 67-73. 
[25] Lim, T.-C. On fixed point stability for set-valued contractive mappings with applications to generalized differential equations, J. Math. Anal. Appl. 110 (1985) 436-441.

[26] Loridan, P. Necessary conditions for E-optimality. In: Guignard M. (eds) Optimality and Stability in Mathematical Programming. Mathematical Programming Studies, vol 19. Springer, Berlin, Heidelberg (1982).

[27] Mansour, M.A., Bahraoui, M. A. and El Bekkali, A. Metric regularity and Lyusternik-Graves theorem via approximate fixed points of set-valued map in noncomplete metric spaces, Set-Valued Var. Anal (2020).

[28] NadlerJr., S.B. Multi-valued contraction mappings, Pac. J. Math. 30 (1969) 475-488.

[29] Nashine, H.K., Agarwal, R.P. and Kadelburg, Z. $\mathcal{H}^{+}$-multivalued contractions and their application to homotopy theory, J. Fixed Point Theory Appl. 19 (2017) 2309-2325.

[30] Pacurar, M. and Pacurar, R.V. Approximate fixed point theorems for weak contractions on metric spaces, Carpathian J. Math. 23 (2007) 149-155.

[31] Petrusel, A., Petrusel, G. and Yao, J-C. Pseudo-contractivity and Metric Regularity in Fixed Point Theory, J. Optim. Theory Appl. 180 (2019) 5 -18.

[32] Petrusel, A., Petrusel, G. and Yao, J-C. Multi-valued graph contraction principle with applications, Optimization, 69 (2020) 1541-1556.

[33] Reich, S. Approximate selections, best approximations, fixed points, and invariant sets. J. Math. Anal. Appl. 62 (1978) 104113.

[34] Tijs, S., Torre, A. and Brânzei, R. Approximate fixed point theorems, Libertas Math, 23 (2003) 35-39 .

[35] Tijs, S. Nash equilibria for non-cooperative n-person games in normal form, SIAM Review, 23(1981) 225 - 237. 